Article

\title{
Assessment of Performance of Two Rapid Methods for On-Site Control of Microbial and Biofilm Contamination
}

\author{
Fernando Lorenzo *, Maria Sanz-Puig, Ramón Bertó and Enrique Orihuel \\ Betelgeux-Christeyns, 46729 Valencia, Ador, Spain; m.sanz@betelgeux.es (M.S.-P.); r.berto@betelgeux.es (R.B.); \\ e.orihuel@betelgeux.es (E.O.) \\ * Correspondence: f.lorenzo@betelgeux.es
}

Received: 3 December 2019; Accepted: 16 January 2020; Published: 21 January 2020

check for updates

Featured Application: Validation of hygiene processes in food industries.

\begin{abstract}
Background: The validation of hygiene procedures in food industries is paramount to ensure that food contact surfaces are properly decontaminated before production. Rapid, sensitive and reliable tools are needed for routine hygiene validation in order to increase food safety levels. Two novel tools for biofilm detection (TBF 300) and detection of low levels of microbial contamination (FreshCheck) have been assessed. (2) Methods: Biofilms of relevant food pathogens: Listeria monocytogenes and Salmonella spp. were grown for 3 and 10 days to assess the performance of the biofilm detection product. Surfaces were inoculated with different levels of L. monocytogenes to determine the limit of detection of FreshCheck. (3) Results: TBF 300 visibly stained 3 days-old biofilms of both pathogens, containing 5.0-5.4 $\log \mathrm{CFU} / \mathrm{cm}^{2}$. FreshCheck showed a positive reaction with contamination levels as low as $10 \mathrm{CFU} / \mathrm{cm}^{2}$ for L. monocytogenes. (4) Conclusions: Assessment of the hygienic status of food contact surfaces before production can be greatly improved with the use of the two novel tools evaluated in this study. The detection of microorganisms' presence at very low levels of contamination as well as identification of biofilm growth spots is available in a rapid and easy way, with a big potential contribution to food safety.
\end{abstract}

Keywords: food safety; hygiene; microbial contamination; biofilms; rapid detection

\section{Introduction}

In food production, the presence of unwanted microorganisms is a major risk for the quality and safety of the food products. Microorganisms in the food production environment can contaminate foodstuffs and cause spoilage or foodborne illnesses when ingested. Bacteria such as Listeria monocytogenes, Salmonella enterica subesp. enterica serovar Typhimurium, Campylobacter spp. or Escherichia coli OH157:H7 are major causes of contamination outbreaks and foodborne illnesses [1,2]. A total of 5079 foodborne outbreaks were reported in the European Union in 2017 [3]. Hygiene is essential in preventing microbial contamination of food, working surfaces and equipment. Cleaning and disinfection is thus an important part of the operation of food industries. Improper hygiene can lead to remaining food residues on work surfaces and equipment [4]. This has two detrimental effects: (i) partial inactivation of disinfectants used for decontamination of surfaces, resulting in limited decontamination and potential exposure of microorganisms to sublethal concentrations of biocide $[5,6]$; and (ii) presence of anchor points for the attachment of bacteria, favoring the growth of a biofilm. In both cases, the result is the occurrence of persistent contamination [7], comprised of microorganisms that resist conventional hygiene procedures, either because they have developed increased tolerance to 
the biocides employed or because they are protected by a biofilm [8-10]. Persistent microorganisms can cause long-term contamination of the food production plant, potential outbreaks of food pathogens and ultimately, even lead to closure of the factory [11].

Hygiene procedures are usually aimed at removing physical, chemical and microbial contamination from the production environment [12] and should be based on a risk assessment program where the critical contamination points, sources of contamination and target levels to ensure food safety should be established. Validation of the hygiene process is therefore of great importance to check that cleaning and disinfection has been done correctly and that food contact surfaces and production equipment are free of contamination before production. Several tools and methodologies are currently available for this purpose, with different scopes for detection of contamination and variable features in terms of cost, ease of use, speed, and specificity [13]. The most commonly used techniques are summarized in Table 1.

Table 1. Summary of techniques and methodologies currently employed for validation of hygiene in food production environments.

\begin{tabular}{|c|c|c|c|c|}
\hline Technique & Target for Detection & Cost per Sample * & Ease of Use ${ }^{\dagger}$ & Specificity $\ddagger$ \\
\hline ATP bioluminescence & ATP molecules contained in living cells & Moderate & High & Moderate \\
\hline Contact plating & Viable microorganisms & Low & High & High \\
\hline Swabbing & Viable microorganisms & High & Low & High \\
\hline Test strips & Residues of detergents or disinfectants & Low & High & High \\
\hline Visual inspection & Visible residual soiling & Low & High & Low \\
\hline
\end{tabular}

${ }^{*}$ Low: no or inexpensive materials $(<1 € /$ sample) required. Moderate: low cost for fungibles $(<3 € /$ sample) but expensive equipment required. High: Dedicated facilities and personnel required. ${ }^{\dagger}$ Low: requires personnel with specific training. High: Basic training required. $\ddagger$ Low: Limited discrimination of contamination types. Moderate: Discrimination between organic/biologic contamination and inorganic contamination. High: A specific type of contamination is detected.

The detection of microbial contamination is usually limited by the lack of specificity of the tools available. For example, measurement of ATP luminescence is often employed for this purpose due to the presence of ATP molecules in every microorganism, but this is also the case of other cells present in food residues [14]. Additionally, microorganisms surviving a disinfection process can be stressed and have their ATP levels depleted [15], leading to underestimation of microorganism numbers when using this technique. Specific methods for detection of microorganisms, such as contact plates or swabbing provide information on the numbers of microorganisms recovered from the surfaces and, if selective media are used, they will also discriminate among different classes of microorganisms. On the other hand, these methods require incubation of samples, sometimes preceded by an enrichment step [16]. For this reason, selective detection of microorganisms usually requires $18-72 \mathrm{~h}$ for most bacteria and up to 5 days for fungi. Finally, visual inspection is an essential part of the hygiene audit, since it allows identifying bad hygiene practices, lack of equipment maintenance, improper use of equipment, and other aspects of the hygiene procedure. However, this requires experienced personnel and a systematic approach to provide useful results.

Novel methods for the detection of microbial contamination are needed to improve the validation of hygiene procedures in food industries and others where the presence of microorganisms is of concern. Ideally, these methods should be (i) fast, so that they can be used on site after cleaning and disinfection and before production resumes; (ii) easy to use, so that they do not require specialized personnel or equipment; (iii) non-expensive, accessible to industries of all sizes; and (iv) specific, so that more detailed information on the characteristics of the contamination can be obtained.

In particular, detection of biofilms is critical due to the potential risk for food safety in production environments [17]. Conventional microbial sampling techniques such as swabbing or ATP bioluminescence do not provide information on the presence of a biofilm. Confirmation of the presence of a microbial biofilm usually requires microscopic techniques, which are not useful for routine controls. In the past few years, there has been growing interest in the use of dye-based reagents to stain the biofilm matrix and thus reveal their presence [18]. Among these, $\mathrm{TBF}^{\circledR} 300$ (Betelgeux-Christeyns, 
Valencia, Spain) has been available in the market since 2011 as a rapid tool for specific identification of biofilms $[18,19]$. TBF ${ }^{\circledR} 300$ is based on Rhodamine B as a staining agent, which is a counterstaining dye and is reported to reveal the total extent of biomass, independent of its activity [20]. This product is applied as foam or sprayed onto sample points, where it is allowed to act for a few minutes and then rinsed. The composition of TBF 300 includes surfactants and auxiliary components to enhance foam performance and stability of the dye. The permanence of residual staining after the washing step is normally associated to the presence of biofilms on the surface [21,22]. This product is recommended for application on metallic surfaces due to the risk of permanent staining of porous or some plastic surfaces, which would lead to false positives.

A more recent tool of great interest for assessing the hygiene status of surfaces is FreshCheck (Fresh Check UK). This product is based on Chromeazurol S (CAS), a chromophore containing iron $\left(\mathrm{Fe}^{3+}\right)$ that changes its color when the iron ion is removed from its structure [23]. Most microorganisms produce siderophores with high iron binding affinity, so they will sequester iron from CAS and thus produce a visible change in color [24]. The change in color can also occur in the presence of other residues usually found in the food industry, such as residual detergents or food soiling [25]. FreshCheck is sprayed onto test surfaces and results are obtained within $30 \mathrm{~s}$, in the form of a color change easily visible with the naked eye. FreschCheck is purple when applied on clean surfaces and it changes to a range of colors (usually green or orange) when reacting with residual contamination.

These novel tools represent a valuable opportunity for food producers and hygiene auditors to enhance the verification process. Their use can help to obtain greater detail on the presence of contamination after cleaning and disinfection and its nature, in a rapid and easy way that facilitates routine checking of hygiene conditions. However, in order for them to be useful, more information on their performance is required, especially in regards to the sensitivity and accuracy of the results obtained. In this study, the performance of TBF 300 for detection of biofilms formed by L. monocytogenes or S. Typhimurium was assessed at two stages of biofilm development: 3 and 10 days. Likewise, the ability of FreshCheck to react to the presence of L. monocytogenes or S. enterica cells was tested and the limit of detection of L. monocytogenes cells was investigated.

The use of dyes to stain food residues or microbial contamination in the form of biofilms has been extensively reported. However, most of the studies published focus on the use of dyes for laboratory application or other types of biofilms (oral biofilms for example) [26]. There are very few examples of evaluation of the use of dye-based products for validation of hygiene in food industries, looking at their performance in conditions representative of the food industry [27]. The products reported in this study are novel in the food hygiene sector as final, ready-to-use products, and their evaluation in the context of their practical contribution to validation of hygiene processes has not been previously reported in a scientific way.

\section{Materials and Methods}

\subsection{Bacterial Cultures and Growth Conditions}

Pure cultures of L. monocytogenes (ATCC 07644) and S. enterica (ATCC 13076) were provided freeze-dried by the American Type Culture Collection. Stocks of each freeze-dried microorganism were obtained and $2 \mathrm{~mL}$ samples were stored in freezing. A cryovial of each microorganism was defrosted and rehydrated with $50 \mathrm{~mL}$ of Tryptic Soy Broth (TSB) (Scharlab Chemie, Barcelona, Spain) and incubated overnight at $37^{\circ} \mathrm{C}$ to reach its exponential growth phase.

\subsection{Biofilm Growth}

Biofilms were grown on AISI 304 stainless steel plaques as supporting material (sizes $45 \times 45 \mathrm{~mm}$ and $90 \times 90 \mathrm{~mm}$ ), representative of food contact surfaces in production environments. Surfaces for biofilm growth were divided into three groups:

- Clean and sterile stainless steel surfaces where monospecies and mixed biofilms are grown. 
- Clean and sterile stainless steel surfaces, without microbial contamination, used as control

- Clean and sterile stainless steel surfaces where planktonic cells are inoculated to show the presence of biofilms without biofilm formation.

Generic, non-selective culture media were employed for growing and forming biofilms: TBS (Typtic Soy Broth, Scharlab, Barcelona, Spain) and BHI (Brain Heart Broth, Merck, Madrid, Spain). Working temperature was $37^{\circ} \mathrm{C} \pm 1{ }^{\circ} \mathrm{C}$, which is the optimal growth temperature for the microorganisms tested.

Biofilms were grown under static and dynamic conditions $(11.6 \mathrm{~mL} / \mathrm{min})$, with and without replenishment of nutrition media, in order to optimize microbial adhesion to surfaces. Two biofilm growth periods, 3 and 10 days, were considered for testing TBF 300 against biofilms with different levels of strength.

\subsection{Staining of Biofilms}

Once biofilms were formed, surfaces were washed with distilled water to remove planktonic cells (not adhered to the surface) and allowed to dry in a laminated flow cabinet. Afterwards, the staining agent was applied and allowed to act for $15 \mathrm{~min}$. Then, surfaces were rinsed with distilled water ( 2 washes during $10 \mathrm{~s}$ each) and were examined to reveal the permanence of staining.

The efficacy of staining was assessed through the observation of colored spots on surfaces after rinsing and through inspection of surfaces under UV irradiation (lamp emitting at 254 and $366 \mathrm{~nm}$ ). Application tests were performed in triplicate for each microbial group (biofilms and planktonic cells) to ensure results were representative.

Non-contaminated surfaces were used and controlled in staining tests in order to assess the selectivity of TBF 300. Additionally, surfaces contaminated with planktonic cells were also used as a secondary control.

\subsection{Bacterial Counts and Determination of Biomass FreshCheck Studies}

FreshCheck sensitivity to detect microbial contamination on surfaces was assessed before and after the application of different biocidal products. For this purpose, stainless steel plates were inoculated with L. monocytogenes and S. enterica at high concentrations (approximately $10^{7} \mathrm{CFU} / \mathrm{mL}$ ) using a cotton swab and then FreshCheck was applied.

Subsequently, different biocidal products were applied on these surfaces for $5 \mathrm{~min}$ in order to eliminate the microbial load. After that, each biocide was rinsed with water and then FreshCheck was applied again. Table 2 shows the biocide active substance in each of the products tested. All biocides were supplied by Betelgeux-Christeyns:

Table 2. Active biocide substances in each tested product.

\begin{tabular}{ccc}
\hline Product Number & Product Name & Main Biocide Substance \\
\hline 1 & Dectocide A30 & Alkylamine \\
2 & Dectocide SB9 & Glutaraldehyde \\
3 & Dexacide B10 & Benzalkonium chloride \\
4 & Quacide PQ60 EC & Alkylamine + PHMB \\
5 & Quacide DA80 & Lactic acid \\
\hline
\end{tabular}

Finally, the capacity of FreshCheck to detect decreasing levels of microbial contamination was evaluated in comparison with contact slides, a tool usually used to evaluate hygiene on food industry surfaces. Starting from an initial solution of $10^{7} \mathrm{CFU} / \mathrm{mL}$ of L. monocytogenes, essay solutions were obtained with different microbial concentrations: $10^{6}, 10^{5}, 10^{4}, 10^{3}, 10^{2}$, and $10^{1} \mathrm{CFU} / \mathrm{mL}$, by serial dilution. Each of these solutions were applied on sterile stainless steel surfaces using a cotton swab. Immediately, each surface was sampled with contact slides and tested with the FreshCheck spray. 


\section{Results}

\subsection{Biofilm Staining}

Biomass concentration values for biofilms formed on stainless steel surfaces after 3 and 10 days are shown in Tables 3 and 4 and visually represented in Figure 1.

Table 3. Biomass values for biofilms after 3 days growth.

\begin{tabular}{ccccc}
\hline & \multicolumn{3}{c}{ 3 Days Biofilm } \\
\hline Microorganism & $\begin{array}{c}\text { Biomass } \\
\text { (CFU/surface) } \mathbf{n}=\mathbf{3}\end{array}$ & $\begin{array}{c}\text { Biomass } \\
\left(\mathbf{C F U} / \mathbf{c m}^{\mathbf{2}}\right) \mathbf{n}=\mathbf{3}\end{array}$ & $\begin{array}{c}\text { Biomass (log } \\
\text { CFU/surface) } \mathbf{n}=\mathbf{3}\end{array}$ & $\begin{array}{c}\text { Biomass (log } \\
\left.\mathbf{C F U} / \mathbf{c m}^{\mathbf{2}}\right) \mathbf{n}=\mathbf{3}\end{array}$ \\
\hline Salmonella spp. & $2.8 \times 10^{6}$ & $1.6 \times 10^{5}$ & $6.45 \pm 0.70$ & $5.2 \pm 0.56$ \\
L. monocytogenes & $4.2 \times 10^{6}$ & $2.3 \times 10^{5}$ & $6.62 \pm 0.04$ & $5.4 \pm 0.03$ \\
Salmonella spp. + L. monocytogenes & $1.6 \times 10^{6}$ & $8.9 \times 10^{4}$ & $6.21 \pm 0.25$ & $5.0 \pm 0.20$ \\
\hline
\end{tabular}

Table 4. Biomass values for biofilms after 10 days growth.

\begin{tabular}{ccccc}
\hline & & \multicolumn{2}{c}{ 10 Days Biofilm } \\
\hline Microorganism & $\begin{array}{c}\text { Biomass } \\
\text { (CFU/surface) } \mathbf{n}=\mathbf{3}\end{array}$ & $\begin{array}{c}\text { Biomass } \\
\left(\mathbf{C F U} / \mathbf{c m}^{\mathbf{2}}\right) \mathbf{n}=\mathbf{3}\end{array}$ & $\begin{array}{c}\text { Biomass (log } \\
\text { CFU/surface) } \mathbf{n}=\mathbf{3}\end{array}$ & $\begin{array}{c}\text { Biomass }(\mathbf{l o g} \\
\left.\mathbf{C F U} / \mathbf{c m}^{\mathbf{2}}\right) \mathbf{n}=\mathbf{3}\end{array}$ \\
\hline Salmonella spp. & $6.2 \times 10^{6}$ & $3.5 \times 10^{5}$ & $6.79 \pm 0.51$ & $5.5 \pm 0.42$ \\
L. monocytogenes & $1.8 \times 10^{7}$ & $9.7 \times 10^{5}$ & $7.24 \pm 0.93$ & $6.0 \pm 0.77$ \\
Salmonella spp. + L. monocytogenes & $7.3 \times 10^{6}$ & $4.0 \times 10^{5}$ & $6.86 \pm 0.13$ & $5.6 \pm 0.11$ \\
\hline
\end{tabular}

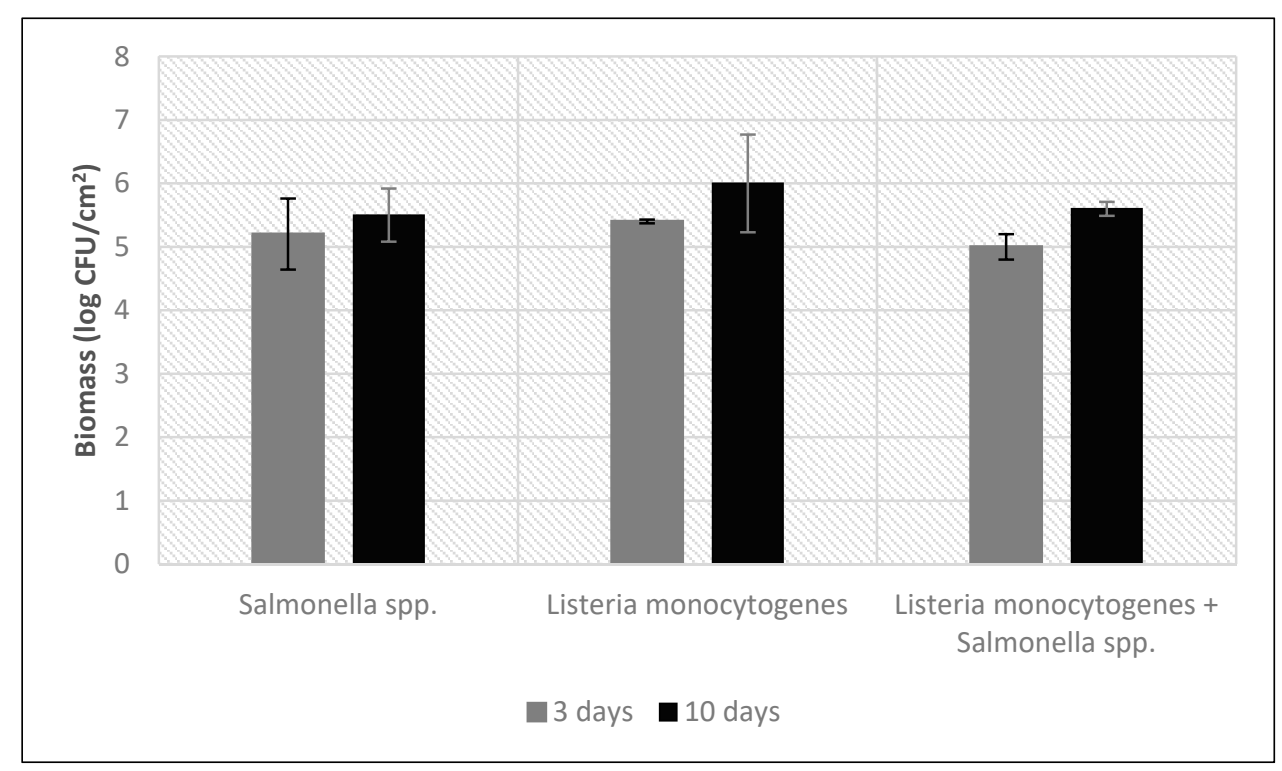

Figure 1. Biomass of biofilms after $3 \square$ and 10 days $\mathbf{m}$, expressed as $\log \mathrm{CFU} / \mathrm{cm}^{2}$.

Microscopic examination of the inoculated coupons shows that microorganisms were able to form biofilms on work surfaces up to $10^{6} \mathrm{CFU} /$ surface (Figure 2). 

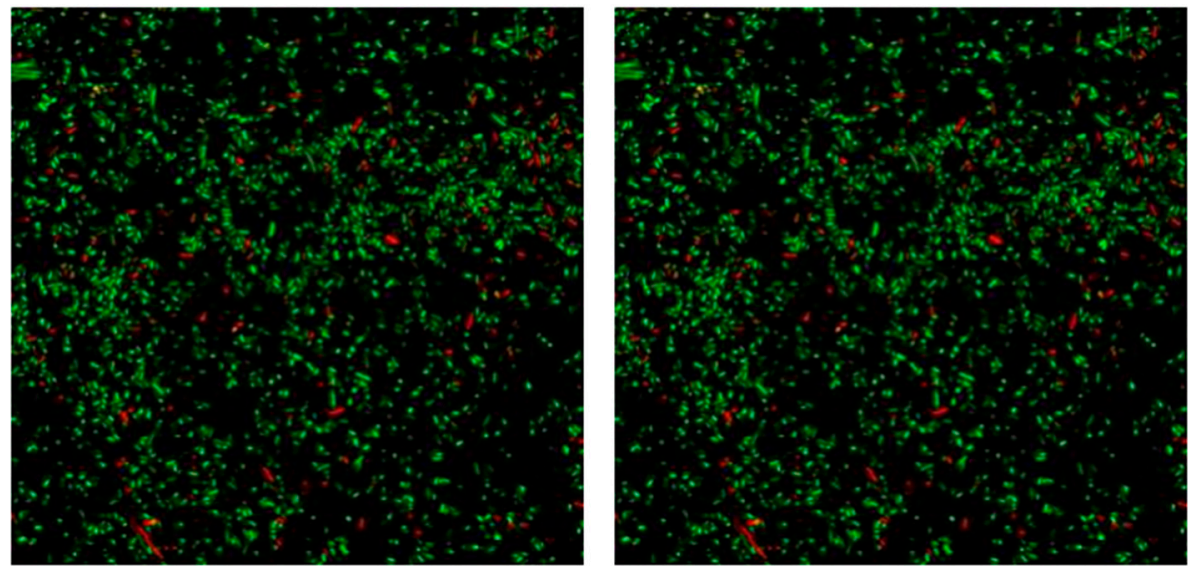

Figure 2. Microscopic image of L. monocytogenes (left) and Salmonella spp. (right) biofilms after LIVE/DEAD ${ }^{\circledR}$ BacLight staining and capture by confocal laser microscopy (60X magnification).

Following optimization of biofilm growing conditions, biofilm staining tests were performed using the TBF 300 product. Both stable, strongly adhered biofilms and non-stable biofilms showing a smaller amount of biomass were stained. The product was applied as a foam over the test surface, covering the whole surface with a layer approximately 3-mm thick. A contact time of $15 \mathrm{~min}$ was allowed, followed by rinsing. The process is illustrated in Figure 3.
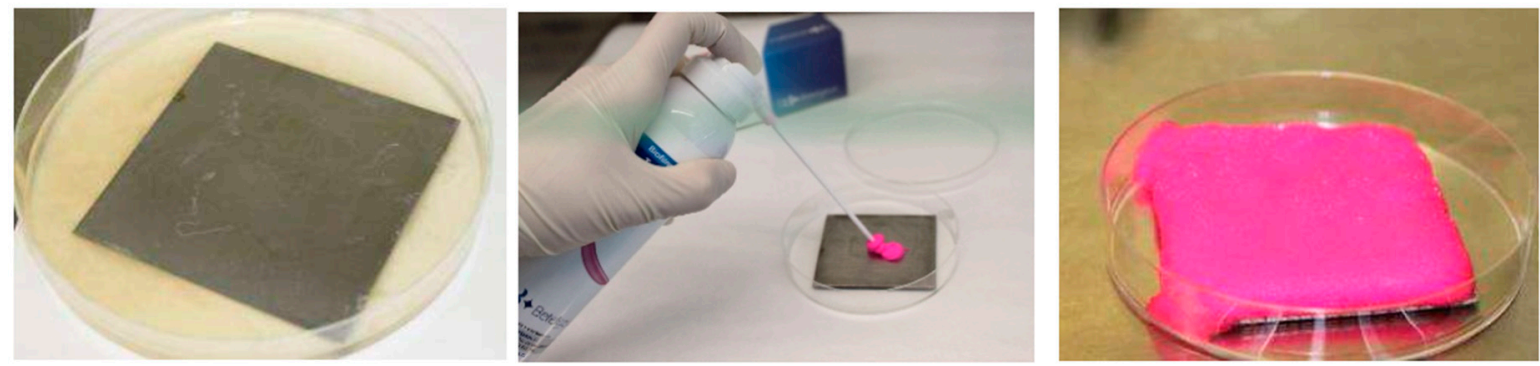

Figure 3. Images showing the procedure for application of TBF 300 for biofilm staining, followed by rinsing and observation of residual staining.

Staining of biofilms grown over 3 days was found to be low, reflecting the fact that adherence of cells in these conditions is weak and the biofilm formed is removed from the surface with relative ease during the washing step. In this case, residual staining was only found in those areas where adhesion of microorganisms to steel is stronger, probably due to the presence of cracks or crevices. This occurs especially in edges and cutting points (see Figure 4).
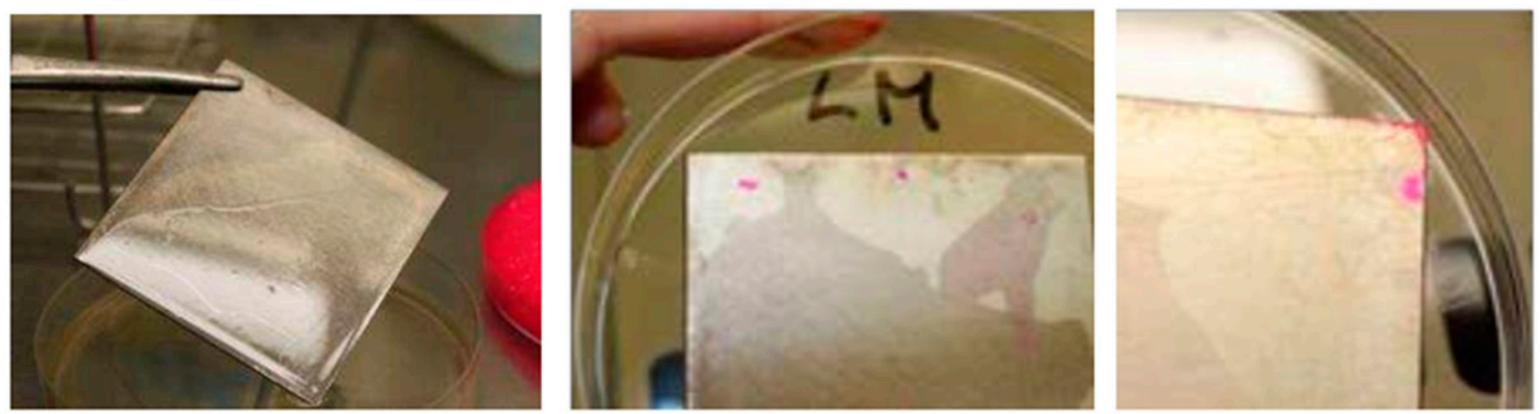

Figure 4. Residual staining after application of TBF 300 on a coupon where a L. monocytogenes biofilm was grown for 3 days. 
Biofilms grown for 10 days showed stronger residual staining after the washing step. These biofilms are expected to contain a higher amount of biomass and show a stronger adherence to surfaces, hence allowing for increased visibility after staining (Figure 5).
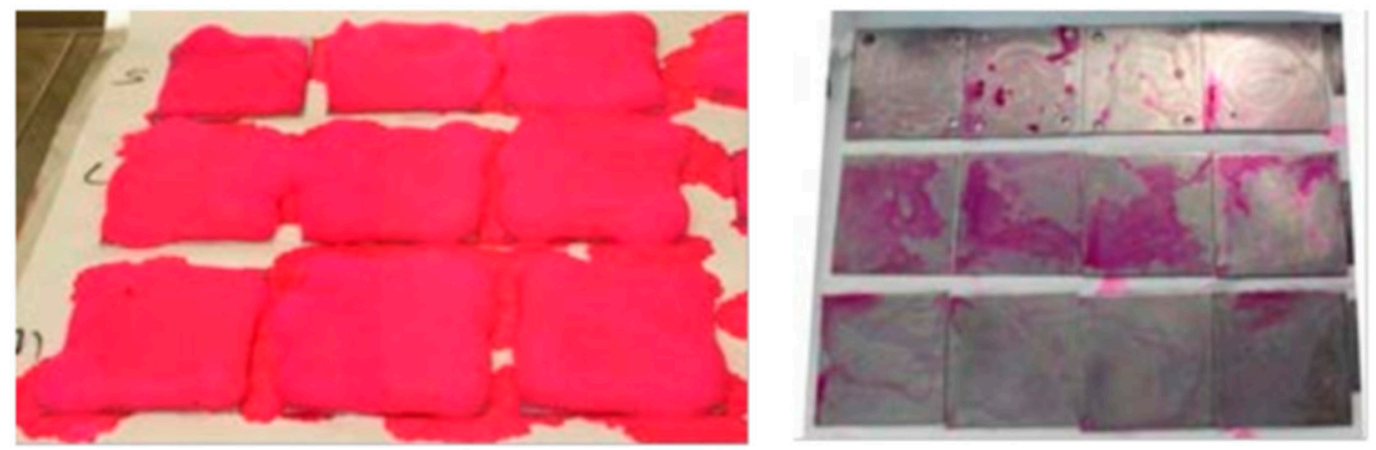

Figure 5. Residual staining after the application of TBF 300 on coupons where microbial biofilms were grown for 10 days. Each row corresponds to biofilms of different microorganisms: Salmonella spp. (upper row), L. monocytogenes (central row), mixed biofilm (lower row).

Coupons containing biofilm were also examined under UV light after staining with TBF 300 and washing. The dye contained in the product shows fluorescent properties under UV irradiation, which was shown to provide a better way for discrimination of biofilm-containing surfaces, as shown in Figure 6.
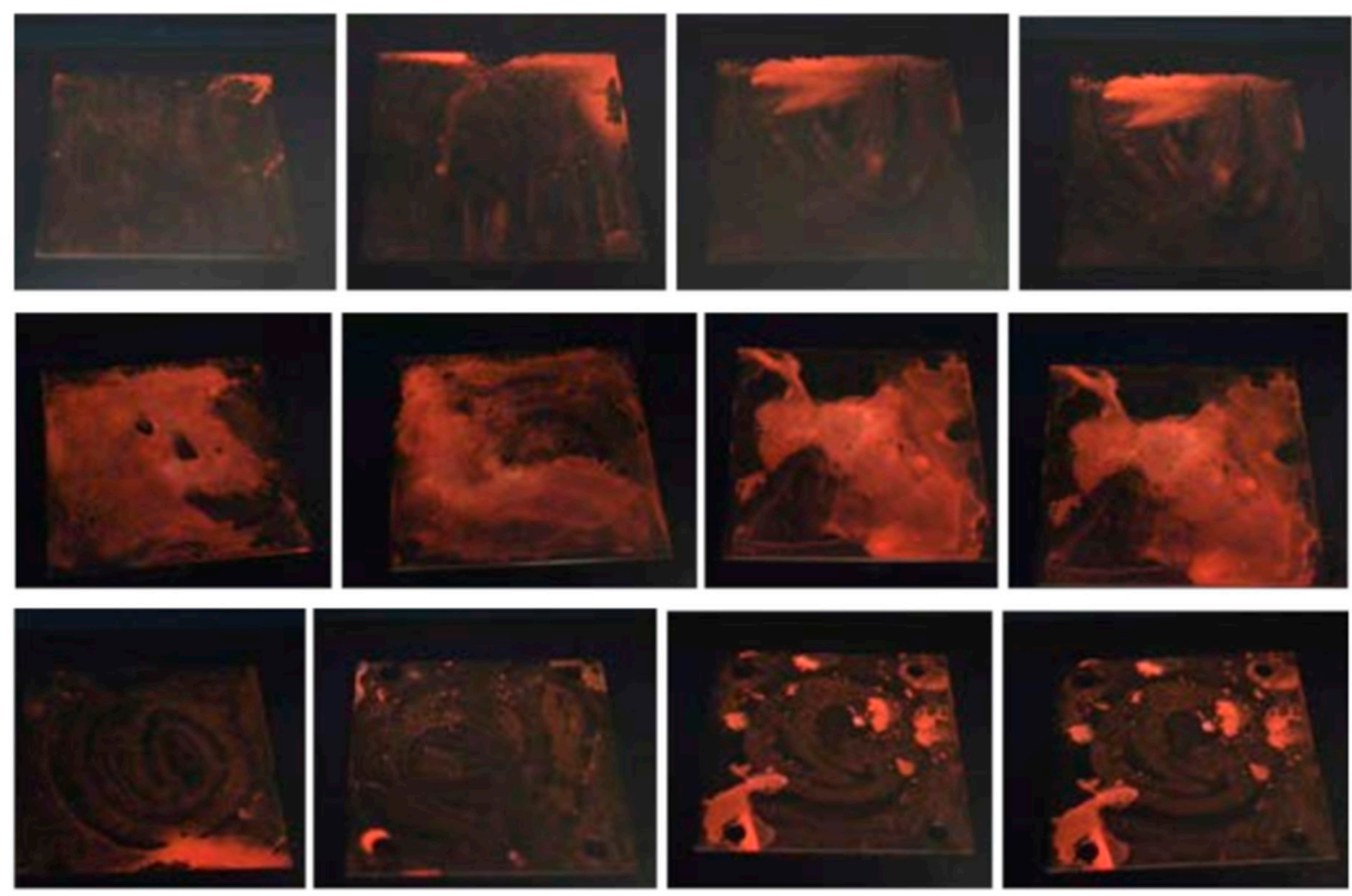

Figure 6. Biofilm (10 days old) containing coupons under UV irradiation. Each row corresponds to biofilms of different microorganisms: Salmonella spp. (upper row), L. monocytogenes (central row), mixed biofilm (lower row).

These results show that TBF 300 can reliably reveal biofilm presence on stainless steel surfaces after its application in the described conditions. Staining intensity is greater as the age of the biofilm 
increases, as a result of a higher amount of matrix in the biofilm. In order to assess the specificity of TBF 300 for biofilm detection, the product was also applied on surfaces inoculated with planktonic cells, without biofilm formation. Staining tests were also performed on control surfaces not inoculated with microorganisms. These tests show that after $15 \mathrm{~min}$ contact time and a rinse step, no dye remains on the coupon surface and neither fluorescence is observed under UV light (Figure 7).
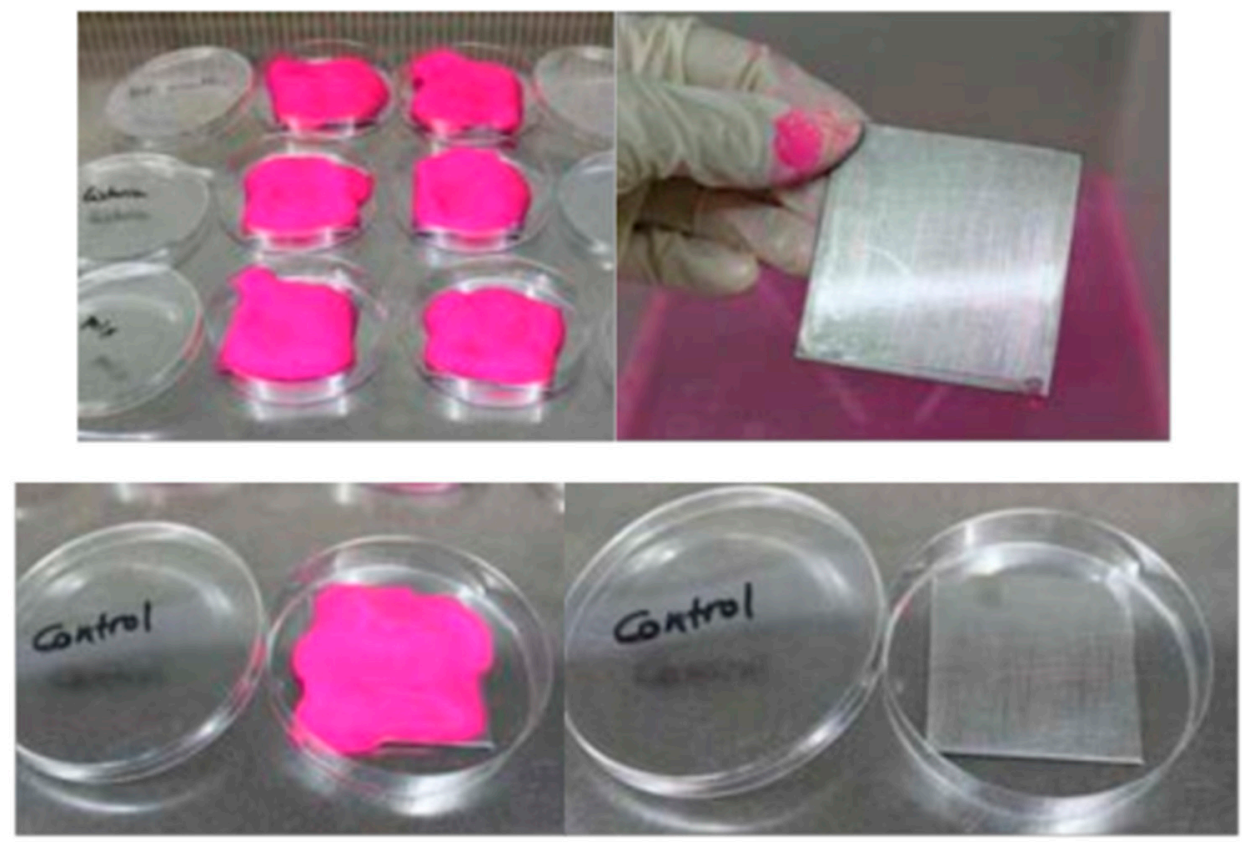

Figure 7. TBF 300 applied on stainless steel coupons inoculated with the test microorganisms without biofilm formation (upper) and non-inoculated (lower). After 15 min contact time and washing, no residual staining is observed.

\subsection{Microbial Detection Using FreshCheck}

Results obtained with FreshCheck before and after the application of different biocides against L. monocytogenes and S. enterica are shown in Table 5.

Table 5. Color observed after application of FreshCheck on surfaces inoculated with L. monocytogenes or S. enterica, before and after disinfection.

\begin{tabular}{cccc}
\hline Biocide & Before Disinfection (Both Microorganisms) & $\begin{array}{c}\text { After Disinfection } \\
\text { (L. monocytogenes) }\end{array}$ & $\begin{array}{c}\text { After Disinfection } \\
\text { (S. enterica) }\end{array}$ \\
\hline 1 & Green & Purple & Purple \\
2 & Green & Purple & Purple \\
3 & Green & Purple & Purple \\
4 & Green & Purple & Purple \\
5 & Green & Purple & Purple \\
\hline
\end{tabular}

As shown in Table 5, in all cases, FreshCheck changed to green when it was tested before the application of each biocide studied, showing the microbial contamination of L. monocytogenes and S. enterica, respectively. In contrast, in all cases the FreshCheck spray remained purple after the application and rinse of all biocides, revealing the absence of microbial charge and confirming that all tested biocides are able to completely inactivate the microbial contamination and that no signal is obtained after successful disinfection. A sample image of the stained coupon can be seen in Figure 8 . 

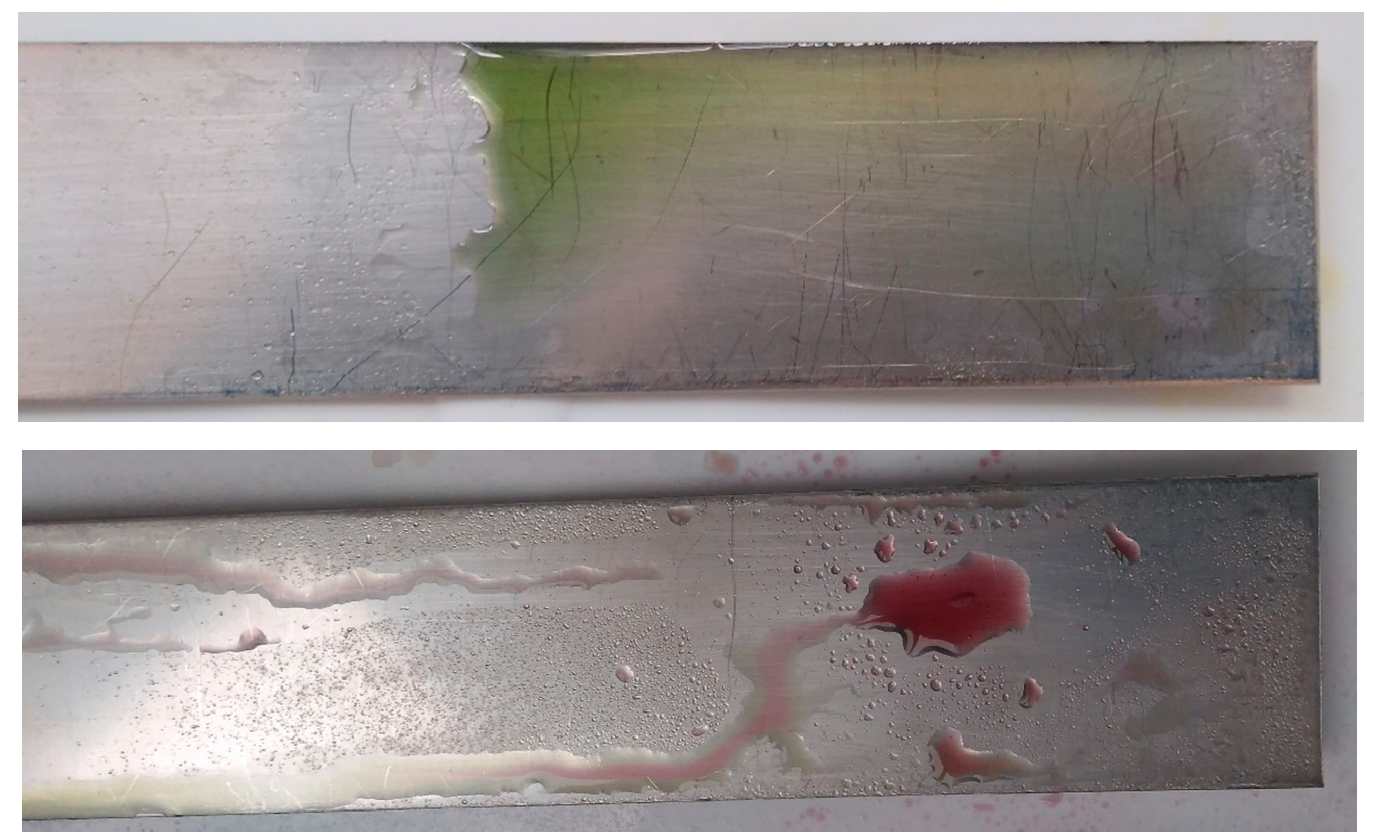

Figure 8. Surfaces after application of FreshCheck showing a negative result (upper, no contamination) and a positive result (lower, presence of microorganisms).

FreshCheck was applied on stainless steel coupons containing different levels of L. monocytogenes contamination, after inoculation with serial dilutions of a suspension containing $10^{7} \mathrm{CFU} / \mathrm{mL}$. Slide contact plates were also applied on a parallel experiment to compare the sensitivity of both methods for detection of microbial contamination. Table 6 shows the results of the comparative study between FreshCheck and contact slides, detecting different concentrations of L. monocytogenes contamination.

Table 6. FreshCheck capacity to detect different levels of microbial contamination of L. monocytogenes in comparison with Contact Slide.

\begin{tabular}{ccc}
\hline Microbial Concentration (CFU/mL) & Contact Slide & FreshCheck \\
\hline $10^{6}$ & $>300$ & Green \\
$10^{5}$ & $>300$ & Green \\
$10^{4}$ & $>300$ & Green \\
$10^{3}$ & 52 & Green \\
$10^{2}$ & - & Green \\
$10^{1}$ & 2 & Green \\
0 (control) & - & Purple \\
\hline
\end{tabular}

Results of Table 6 show that FreshCheck has a very high sensitivity detecting microbial contamination on surfaces. Contact slides were only to recover bacteria able to form visible colonies from surfaces inoculated with a suspension of at least $10^{3} \mathrm{CFU} / \mathrm{mL}$. In contrast, FreshCheck spray shows a positive reaction at all levels of contamination tested, even at values as low as $10 \mathrm{CFU} / \mathrm{mL}$.

\section{Conclusions}

In this study, two novel tools commercially available for validation of hygiene control in food industries have been evaluated. Both detection of microbial contamination at very low levels and identification of biofilm presence in a rapid and easy way are very significant for microbiological control in food industries. With such tools, a quality supervisor can react immediately to hygiene results and optimize cleaning and disinfection procedures to achieve total elimination of microbial contamination. These new techniques can be of great help to complement current methodologies for 
improved assessment of hygienic status in food industries. Their speed and ease of use allows their use before starting the production processes, contributing to guarantee food safety of food products that reach the market.

TBF 300 was able to visibly stain biofilms at least 3 days old for the microorganisms tested. Cells' attachment to surfaces occurs within a few hours, with mature biofilms starting to be formed between $48-72 \mathrm{~h}$ depending on the environmental conditions $[7,28]$. Therefore, TBF 300 can be used for the identification of spots where hygiene procedures are not effective, allowing contamination to accumulate over time and leading to the formation of microbial biofilms. The identification of such spots reveals deficiencies in the hygiene plan and corrective measures can be planned and implemented accordingly.

Biofilm detection is complemented with the use of FreshCheck for generic identification of residues after cleaning and disinfection. The results of this study show that FreshCheck can react with viable microorganisms at very low levels of contamination, so that the efficacy of the hygiene process can be evaluated on the spot with high sensibility. ATP-bioluminescence methods are usually employed for this purpose because of their sensitivity, speed and ease of use. However, they require purchasing expensive equipment for reading bioluminescence results. In contrast, FreshCheck avoids the need for dedicated equipment and is therefore a cheaper approach that can potentially provide more relevant results in the presence of stressed cells without requiring special equipment.

Research on hygiene validation methodologies continues to advance with the aim of providing faster, easier and more informative tools for detection of contamination in food processing environments. The combined use of these tools can help quality supervisors to continuously improve their control on hygiene procedures, with more information on the characteristics of the contamination found and thus potential sources of contamination and corrective measures. Microbial contamination, in the form of non-attached cells or biofilms, is of high concern for food safety, and most control methods available are either non-specific or require long processing times. The two novel tools evaluated in this study improve the process of hygiene validation and prevention of food contamination.

\section{Patents}

The work reported in this manuscript contributed to obtaining the patent EP2634260A1-Biofilmmarking composition and method for detection of same on surfaces [19].

Author Contributions: All authors conceived and planned the work. M.S.-P. performed the experiments. All authors analyzed and/or interpreted the results. F.L. and M.S.-P. wrote the manuscript. All authors have read and agreed to the published version of the manuscript.

Funding: This research received no external funding.

Conflicts of Interest: The funders had no role in the design of the study; in the collection, analyses, or interpretation of data; in the writing of the manuscript, or in the decision to publish the results.

\section{References}

1. Newell, D.G.; Koopmans, M.; Verhoef, L.; Duizer, E.; Aidara-Kane, A.; Sprong, H.; Opsteegh, M.; Langelaar, M.; Threfall, J.; Scheutz, F.; et al. Food-borne diseases-The challenges of 20 years ago still persist while new ones continue to emerge. Int. J. Food Microbiol. 2010, 139, S3-S15. [CrossRef] [PubMed]

2. Orihuel, E.; Bertó, R.; Canet, J.J.; Lorenzo, F.; Milvaques, A.; Sanz-Puig, M.; Sabater, N. Listeria Monocytogenes in the Meat Industry; Betelgeux, S.L., Ed.; Christeyns Food Hygiene: Ador, Spain, 2019; ISBN 978-84-942180-4-0.

3. EFSA; ECDC. The European Union summary report on trends and sources of zoonoses, zoonotic agents and food-borne outbreaks in 2017. EFSA J. 2018, 16, 262.

4. Lelieveld, H.L.M.; Mostert, M.A.; White, B.; Holah, J.T. Hygiene in Food Processing: Principles and Practice, 2nd ed.; Woodhead Publishing Limited: Cambridge, UK, 2003; ISBN 9781855734661.

5. Holah, J.T.; Lavaud, A.; Peters, W.; Dye, K.A. Future techniques for disinfectant efficacy testing. Int. Biodeterior. Biodegrad. 1998, 41, 273-279. [CrossRef] 
6. Bessems, E. The effect of practical conditions on the efficacy of disinfectants. Int. Biodeterior. Biodegrad. 1998, 41, 177-183. [CrossRef]

7. Magalhães, R.; Ferreira, V.; Brandão, T.R.S.; Palencia, R.C.; Almeida, G.; Teixeira, P. Persistent and non-persistent strains of Listeria monocytogenes: A focus on growth kinetics under different temperature, salt, and $\mathrm{pH}$ conditions and their sensitivity to sanitizers. Food Microbiol. 2016, 57, 103-108. [CrossRef] [PubMed]

8. Bridier, A.; Sanchez-Vizuete, P.; Guilbaud, M.; Piard, J.C.; Naïtali, M.; Briandet, R. Biofilm-associated persistence of food-borne pathogens. Food Microbiol. 2015, 45, 167-178. [CrossRef]

9. Srey, S.; Jahid, I.K.; Ha, S. Do Biofilm formation in food industries: A food safety concern. Food Control 2013, 31, 572-585. [CrossRef]

10. Puga, C.H.; Sanjose, C.; Orgaz, B. Biofilm development at low temperatures enhances Listeria monocytogenes resistance to chitosan. Food Control 2016, 65, 143-151. [CrossRef]

11. Melero, B.; Stessl, B.; Manso, B.; Wagner, M.; Esteban-Carbonero, Ó.J.; Hernández, M.; Rovira, J.; Rodriguez-Lázaro, D. Listeria monocytogenes colonization in a newly established dairy processing facility. Int. J. Food Microbiol. 2019, 289, 64-71. [CrossRef]

12. Gibson, H.; Taylor, J.H.; Hall, K.E.; Holah, J.T. Effectiveness of cleaning techniques used in the food industry in terms of the removal of bacterial biofilms. J. Appl. Microbiol. 1999, 87, 41-48. [CrossRef]

13. Lelieveld, H.; Mostert, T.; Holah, J. Handbook of Hygiene Control in the Food Industry; Woodhead Publishing Limited: Cambridge, UK, 2005; ISBN 9781845690533.

14. Davidson, C.A.; Griffith, C.J.; Peters, A.C.; Fielding, L.M. Evaluation of two methods for monitoring surface cleanliness-ATP bioluminescence and traditional hygiene swabbing. Luminescence 1999, 14, $33-38$. [CrossRef]

15. Bajerski, F.; Stock, J.; Hanf, B.; Darienko, T.; Heine-Dobbernack, E.; Lorenz, M.; Naujox, L.; Keller, E.R.J.; Schumacher, H.M.; Friedl, T.; et al. ATP content and cell viability as indicators for cryostress across the diversity of life. Front. Physiol. 2018, 9, 921. [CrossRef] [PubMed]

16. Jasson, V.; Jacxsens, L.; Luning, P.; Rajkovic, A.; Uyttendaele, M. Alternative microbial methods: An overview and selection criteria. Food Microbiol. 2010, 27, 710-730. [CrossRef] [PubMed]

17. Simões, M.; Simões, L.V.M. A review of current and emergent biofilm control strategies. LWT Food Sci. Technol. 2010, 43, 573-583. [CrossRef]

18. Cadena, M.; Kelman, T.; Marco, M.L.; Pitesky, M. Understanding Antimicrobial Resistance (AMR) Profiles of Salmonella Biofilm and Planktonic Bacteria Challenged with Disinfectants Commonly Used During Poultry Processing. Foods 2019, 8, 275. [CrossRef]

19. Orihuel, E.; Bertó, R.; Lorenzo, F.; López, C.; San Jose, C.; Orgaz, B. Biofilm-marking composition and method for detection of same on surfaces. European Patent EP2634260A1, 4 November 2013.

20. Rani, S.A.; Pitts, B.; Beyenal, H.; Veluchamy, R.A.; Lewandowski, Z.; Davison, W.M.; Buckingham-Meyer, K.; Stewart, P.S. Spatial patterns of DNA replication, protein synthesis, and oxygen concentration within bacterial biofilms reveal diverse physiological states. J. Bacteriol. 2007, 189, 4223-4233. [CrossRef]

21. Lorenzo, F.; Orihuel, E.; Bertó, R.; López, C. Control de la presencia de biofilms en las industrias alimentarias. Aliment. Equipos Tecnol. 2011, 264, 43-47.

22. Lorenzo, F.; Orihuel, E.; López, C.; Catalá, M.; Bertó, R.; Orihuel, E.; San Jose, C.; Orgaz, B. A rapid, easy technique for routine control of biofilms on surfaces. In Proceedings of the 2nd Workshop on Food Safety: Technologies and Innovations Applied to Food Safety, Valencia, Spain, 5-6 July 2012.

23. Bond, A.; Simpson, J.; Peach, R. Colour changing compositions. International Patent Application WO 2018/185486 A1, 11 October 2018.

24. Milagres, A.M.F.; Machuca, A.; Napoleão, D. Detection of siderophore production from several fungi and bacteria by a modification of chrome azurol S (CAS) agar plate assay. J. Microbiol. Methods 1999, 37, 1-6. [CrossRef]

25. Campden BRI. Rapid Methods for Hygiene Determination; Campden BRI: Chipping Campden, UK, 2018.

26. Stiefel, P.; Rosenberg, U.; Schneider, J.; Mauerhofer, S.; Maniura-Weber, K.; Ren, Q. Is biofilm removal properly assessed? Comparison of different quantification methods in a 96-well plate system. Appl. Microbiol. Biotechnol. 2016, 100, 4135-4145. [CrossRef] [PubMed] 
27. Gamble, G.R.; Lawrence, K.C.; Park, B.; Yoon, S.; Heitschmidt, G.W. Food Grade Dye for Assessment of Biofilm Removal from Stainless Steel by Cleaning and Sanitizing Agents. Food Prot. Trends 2019, 39, 442-448.

28. Chmielewski, R.A.N.; Frank, J.F. Biofilm Formation and Control in Food Processing Facilities. Compr. Rev. Food Sci. Food Saf. 2003, 2, 22-32. [CrossRef] 